\title{
Excited state baryon spectroscopy from lattice QCD
}

\author{
Stephen Wallace* ${ }^{\dagger}$ \\ Department of Physics, University of Maryland, College Park, MD 20742, USA \\ E-mail: stevewalephysics.umd.edu
}

\begin{abstract}
Lattice QCD calculations are presented for the spectra of $N^{*}$ excited states with spins up to $J=\frac{7}{2}$. Ambiguities of the standard method of spin identification are shown to be overcome by the use of lattice operators that transform according to $S U(2)$ symmetry restricted to the lattice. Such operators are labeled by their continuum spins. Overlaps of the operators with the states obtained by diagonalizing matrices of correlation functions provide a clear link between continuum spins and lattice states, allowing spins to be identified. Evidence for an approximate realization of rotational symmetry in the $N^{*}$ spectrum is presented. In simulations with $m_{\pi} \geq 392 \mathrm{MeV}$, the low-lying excited states of lattice QCD are found to have the same quantum numbers as the states of $S U(6) \otimes O(3)$ symmetry. The lattice spectra are inconsistent with either a quark-diquark model or parity doubling of states and they suggest that the $J=\frac{1}{2}^{+}$Roper resonance may have a complex structure consisting of contributions from $L=0,1$ and 2 .
\end{abstract}

XXIX International Symposium on Lattice Field Theory

July 10 - 162011

Squaw Valley, Lake Tahoe, California

\footnotetext{
*Speaker.

†or the Hadron Spectrum Collaboration.
} 


\section{Introduction}

Substantial progress has been made in recent years by using lattice methods to solve QCD and, thus, to calculate the spectrum of baryonic and mesonic excited states from first principles.

In this report, we describe a recent solution to the problem of spin identification for baryonic excited states. This development has made it possible to determine patterns in the lattice spectra of $N$ and $\Delta$ excited states that have the same spins and numbers of states as in $S U(6) \otimes O(3)$ symmetry. The lattices and methods used to identify spins are described fully in Ref. [1] and the reader should consult that paper for details about the calculations.

The standard method of spin identification relies on patterns of degenerate states across irreps of the octahedral group in the continuum limit. For half-integer spins one uses the double-covered octahedral group. This method has been found to be ambiguous for two simple reasons. First, there is a high degree of degeneracy in lattice spectra and second, the energies of states are subject to uncertainties owing to fluctuations of the gauge configurations. A typical consequence is that approximately degenerate baryon states in $G_{1}, H$ and $G_{2}$ irreps could indicate a spin $\frac{7}{2}$ state, but it could equally well indicate an accidental degeneracy of a spin $\frac{1}{2}$ state and a spin $\frac{5}{2}$ state. This ambiguous situation is not likely to improve without a new method.

The key developments that have enabled spin identification and, thus, better insight into the lattice spectra are i.) the construction of lattice operators that transform as irreducible representations (irreps) of the $S U(2)$ rotational symmetry restricted to the lattice, and ii.) the use of operator overlaps to tell which operators create which states.

The operators are labeled with a continuum spin quantum number, $J$. They are realized by incorporating combinations of covariant lattice derivatives that transform as orbital angular momenta restricted to the lattice. Combinations of the baryon spin, $\mathrm{S}$, and orbital angular momenta, $\mathrm{L} \leq 2$, are constructed to provide total spins $J \leq \frac{7}{2}$. A similar development for integer spins (meson states) was given in Ref. [2].

In order to be suitable for lattice calculations, operators labeled by continuum spin $J$ must be subduced to lattice operators that transform as irreps of the octahedral group. Because of the symmetries of a cubic lattice, it is the latter operators that provide an orthogonal basis for calculations. Subduction is performed using matrices that provide the change of basis from $S U(2)$ quantum states, labeled as $|J, M\rangle$, to quantum states that are irreps of the octahedral group in the continuum limit labeled as $|\Lambda, r,[J]\rangle$, where $\Lambda$ and $r$ denote the irrep and row while $[J]$ denotes the continuum spin. The matrix elements $\langle J, M \mid \Lambda, r,[J]\rangle$ provide the subduction matrices.

\section{Approximate rotational symmetry and spin identification}

Even though the lattice spacing used in this work is not especially small, good evidence is found for an approximate realization of the continuum rotational symmetry in the lattice spectra. An example of this is given in Fig. 1, which indicates the relative sizes of matrix elements of a $48 \times$ 48 matrix of nucleon correlation functions in the $H_{u}$ irrep of the double-covered octahedral group. The $u$ subscript of $H_{u}$ denotes ungerade, or negative parity. Each pixel indicates the magnitude of one matrix element according to the scale given in the lower right corner. The 48 operators used consist of 28 operators that are subduced from $J=\frac{3}{2}$, whose matrix elements are in the upper 


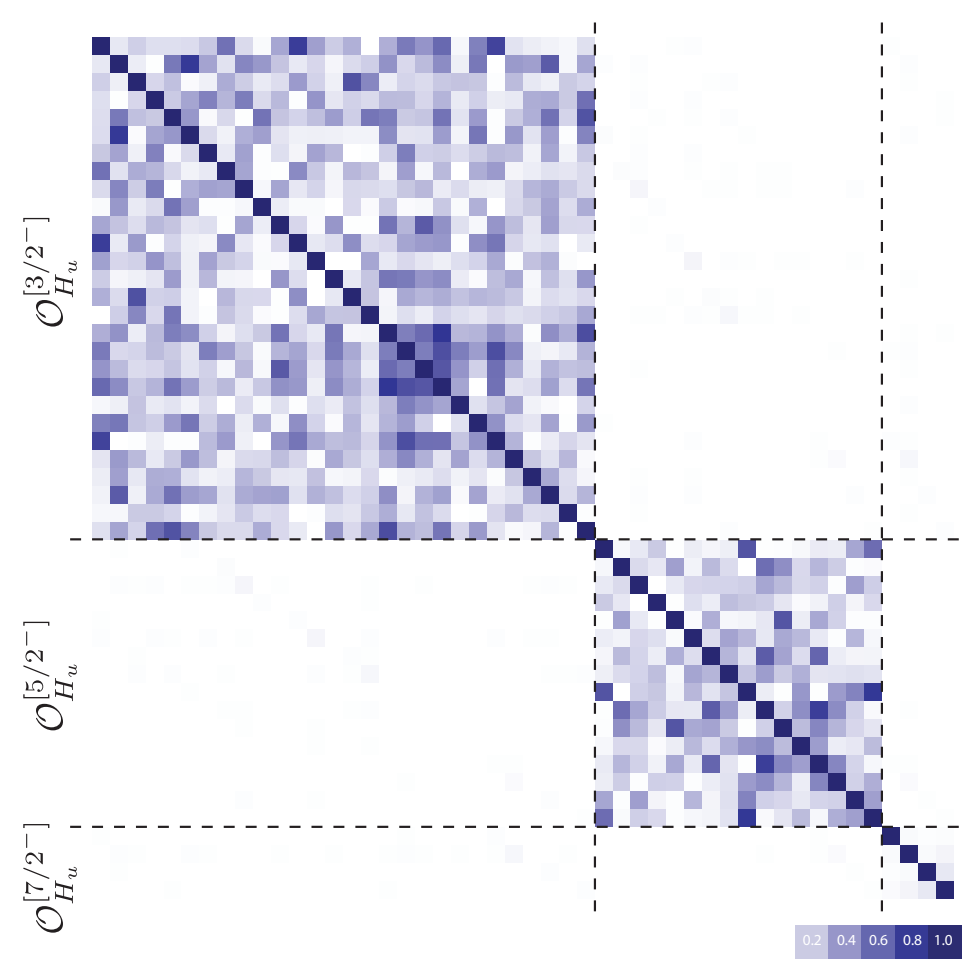

Figure 1: Matrix of $H_{u}$ correlation functions at time slice 5.

block; 16 operators that are subduced from $J=\frac{5}{2}$, whose matrix elements are in the middle block; and 4 operators that are subduced from $J=\frac{7}{2}$ in the lower block. Matrix elements that are outside the blocks involve operators subduced from different $J$ values. They are all quite small. Thus, the matrix is close to block diagonal in spin, $J$, which is a signal of the approximate realization of rotational symmetry.

Approximate rotational symmetry also is clearly evident in spectra that are obtained from matrices of correlation functions. When the matrices are diagonalized, they may be expressed (at large time t) as a spectral decomposition,

$$
C_{i j}(t)=\sum_{n} \frac{Z_{i}^{n *} Z_{j}^{n}}{2 m_{n}} e^{-m_{n} t}
$$

where $i$ and $j$ are labels of the operators $\mathscr{O}_{i}$ and $\mathscr{O}_{j}$ that are involved in the correlation function, the sum is over eigenstates with $m_{n}$ being the mass of the $n^{\text {th }}$ eigenstate and "overlap factors", $Z_{i}^{n} \equiv\left\langle n\left|\mathscr{O}_{i}^{\dagger}\right| 0\right\rangle$ are matrix elements for the $i^{t h}$ operator to create the $n^{t h}$ eigenstate. The masses $m_{n}$ determine the spectrum and the overlap factors show which operators create which states.

A detailed examination of the overlap factors shows that lattice states are created predominantly by operators subduced from a single continuum spin, $J$. We identify the spin of each lattice state as the continuum spin of those operators.

Once spins have been identified, a further test of the approximate rotational invariance in the lattice spectra becomes possible. If indeed the couplings are small between states subduced from different continuum spins, then the omission of such couplings should not much affect the 


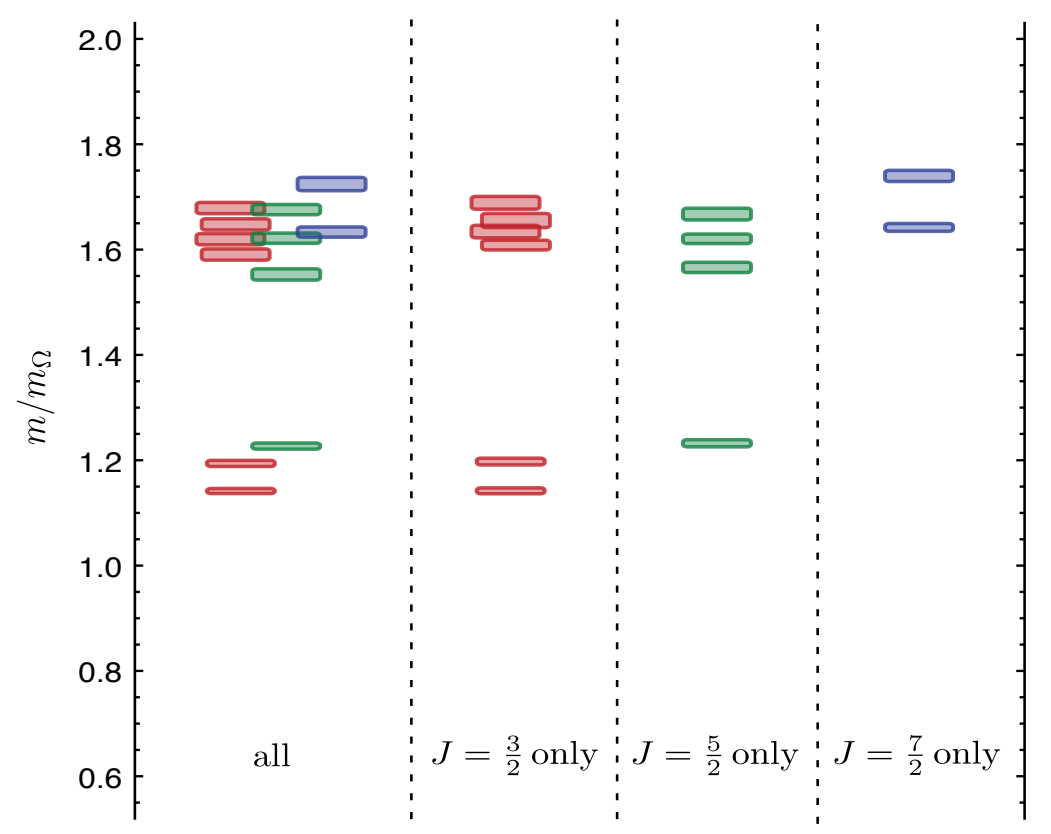

Figure 2: Extracted Nucleon $H_{u}$ mass spectrum for various operator bases. Column 1 uses all $48 H_{u}$ operators, columns 2, 3 and 4 use restricted bases of $28 J=\frac{3}{2}$ operators, $16 J=\frac{5}{2}$ operators and $4 J=\frac{7}{2}$ operators, respectively. States with spin identified as $J=\frac{3}{2}$ are shown in red, $J=\frac{5}{2}$ are shown in green and $J=\frac{7}{2}$ are shown in blue. Results are from the $m_{\pi}=524 \mathrm{MeV}$ ensemble.

excited state spectra. That proposition can be tested by calculating energies using all operators, and comparing them with the energies obtained from the subset of operators subduced from a single $J$ value. If approximate rotational invariance is achieved in the spectrum, the energies should be nearly the same. In Fig. 2, we show such a comparison for the states in the Nucleon $H_{u}$ irrep. The left column of Fig. 2, labelled "all", shows the lowest 12 energy levels obtained from matrices of correlation functions using the set of all $48 H_{u}$ operators, with spins identified using the overlaps, as described above. The second column shows the lowest 6 levels resulting from the variational method when the operators are subduced from continuum spin $J=\frac{3}{2}$. Similarly, the third column shows the lowest 4 levels obtained from the operators subduced from continuum $\operatorname{spin} J=\frac{5}{2}$, and the last column shows the lowest two levels from operators subduced from $J=\frac{7}{2}$.

The results are striking. We see that the masses of the levels in each of the restricted bases agree quite well with the results found in the full basis. The agreement is quite remarkable because one expects that operators in the $H_{u}$ irrep that can couple with the ground state (the lowest $J=\frac{3}{2}$ state), will show a rapid decay of correlators down to the ground state as a function of time, $t$. However, the higher-energy spin $\frac{5}{2}$ and $\frac{7}{2}$ states do not show such a decay; we obtain good fits to correlators at large $t$, where a single mass dominates the decay. These results provide a rather striking demonstration for the lack of significant rotational symmetry breaking in the spectrum.

\section{Lattice $N^{*}$ spectrum with spins identified}

The robust identification of spins allows the spectrum to be displayed as in Fig. 3, with masses 
and uncertainties indicated by boxes in columns labeled by the spin and parity values of the states, $J^{P}$. The excited nucleon spectrum of Fig. 3 contains many more states than the experimental spectrum. All the lattice states, even multiparticle states, are discrete levels (within statistical uncertainties) because of the use of a finite box. Moreover, the use of three-quark operators, as in this work, does not give significant multiparticle contributions, although they should be present in principle. Thus, the expected washing out of highly excited states when they are embedded in a continuum of multiparticle states, does not show up with a small lattice volume. The lattice spectrum is expected to become more realistic with multiparticle operators included in the analyses and volume larger than the present value of $(2 \mathrm{fm})^{3}$.

In Fig. 3, bands of states are indicated by the shaded regions. The lowest band has negative parity states, the next higher band has positive parity states, and as the mass increases, the bands alternate from one parity to the other in a staircase fashion. It has been suggested that a restoration of chiral symmetry might occur at high excitation energy in QCD. [3] That would show up as close to identical masses of higher states in both positive- and negative-parity spectra. The lattice spectra at $m_{\pi}=396 \mathrm{MeV}$ do not provide support for such a parity doubling.

\section{4. $N^{*}$ states with the quantum numbers of $S U(6) \times O(3)$ states}

Focusing on the lowest band of $N^{*}$ states, Fig. 4 shows that the five states have spins based on the combinations of $S=\frac{1}{2}$ and $S=\frac{3}{2}$ with orbital angular momentum and parity $L=1^{-}$. The number of states is tabulated inside the shaded region for each $J$ value and below that we tabulate the construction of spins as $S \oplus L \rightarrow J$. These 5 states belong to the $\left[70,1^{-}\right]$multiplet of $S U(6) \times$ $O(3)$. Although the nucleon operators used are based on four-component Dirac spinors for each quark, the lowest-energy states are created predominantly by operators with only upper components of the Dirac spinors, i.e., Pauli spinors.

The next band at higher mass and positive parity contains $13 N^{*}$ states in the shaded region of Fig. 5, with the number of states listed for each $J$ value. Below that is a tabulation showing the $S \oplus L \rightarrow J$ constructions of the 13 states. The operators used allow the construction to be determined in the same fashion as the spin, i.e., by the overlaps. As can be seen in the tabulation, the 13 states correspond to the positive parity states that can be made by combining $S=\frac{1}{2}$ and $\frac{3}{2}$ with $L=0^{+}, 1^{+}$and $2^{+}$. Nonzero $L$ values are incorporated by covariant derivatives in the operators. If the derivatives were omitted, only one $J=\frac{1}{2}^{+}$state would appear in the shaded region rather than the 4 states that are obtained with derivatives. That is interesting because previous lattice analyses that omit derivative operators generally have found one or two close-together excited $\frac{1}{2}^{+}$ states rather than 4 close-together states, as are obtained here. Our results suggest that the Roper resonance could have a more complex structure that would not be seen without the use of derivative operators. It is an interesting question whether this pattern will still be evident when multiparticle operators are included in the analysis.

It has been suggested that a quark-diquark model might account for the $N^{*}$ spectrum. The lattice results show that the $\left[20,1^{+}\right]$multiplet is realized, i.e., it gives the $5 \mathrm{~L}=1^{+}$constructions seen in the tabulations in Fig. 5. Those states are not consistent with a quark-diquark spectrum. 


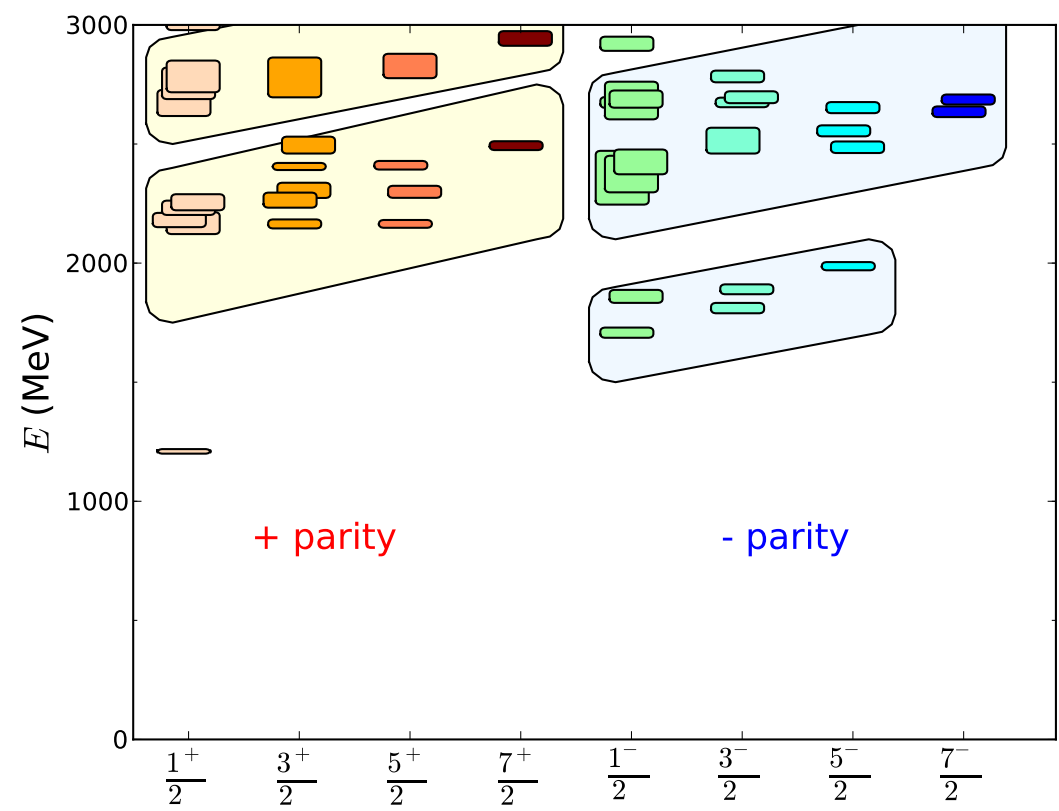

Figure 3: Lattice $N^{*}$ energy spectrum in columns of $\operatorname{good} J^{P}$ at $m_{\pi}=396 \mathrm{MeV}$ with spins identified. Shaded regions show bands of states with alternating parity.

\section{Summary}

The identification of spins of lattice states is significant because the usual method gives ambiguous results, which is a consequence of the high degree of degeneracy in the spectra and uncertainties in the determinations of lattice energies. The use of operators subduced from continuum spins allows robust spin identification. That, in turn, allows the interpretation of low-lying states in the lattice spectra in terms of the quantum numbers of $S U(6) \otimes O(3)$ symmetry. The clarity of the spectrum with spins identified provides contraindications with regard to three oft-discussed issues: parity doubling, quark-diquark models and a simple structure of the $\mathrm{N}^{*} \frac{1}{2}^{+}$(Roper) resonance.

Support from U.S. Department of Energy contract DE-FG02-93ER-40762 is acknowledged. Computations were performed using Chroma [4] and QUDA [5, 6] at Jefferson Laboratory under the USQCD Initiative and LQCD ARRA project.

\section{References}

[1] R. G. Edwards, J. J. Dudek, D. G. Richards and S. J. Wallace Phys. Rev. D 84, 074508 (2011) [ arXiv:1104.5152] .

[2] J. J. Dudek, R. G. Edwards, M. J. Peardon, D. G. Richards, and C. E. Thomas, Phys. Rev. Lett. 103, 262001 (2009) [arXiv:0909.0200].

[3] L. Ya. Glozman, Phys. Lett. B 475, 329-334 [arXiv:hep-ph/9908207].

[4] R. G. Edwards and B. Jóo, Nucl. Phys. B. Proc. Suppl. 140, 832 (2005).

[5] M. A. Clark et al., Comput. Phys. Commun. 181 1517-1528 (2010) [arXiv:0911.3191].

[6] R. Babich, M. A. Clark and B. Jóo, (2010) [arXiv:1011.0024]. 


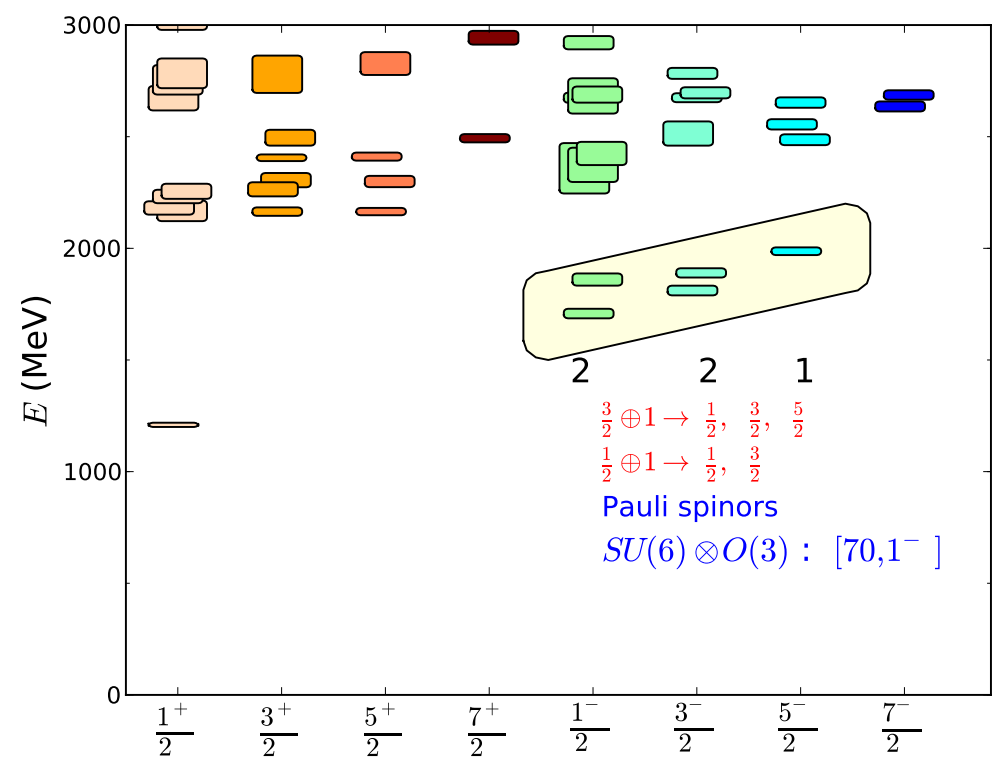

Figure 4: Five $N^{*}$ states corresponding to the $\left[70,1^{-}\right]$multiplet of $S U(6) \times O(3)$ are shown in the shaded area. The $J$ values and parity are the same as can be formed by $S+L \rightarrow J$, as tabulated in red, providing the same numbers of states of each spin as in the lattice spectrum.

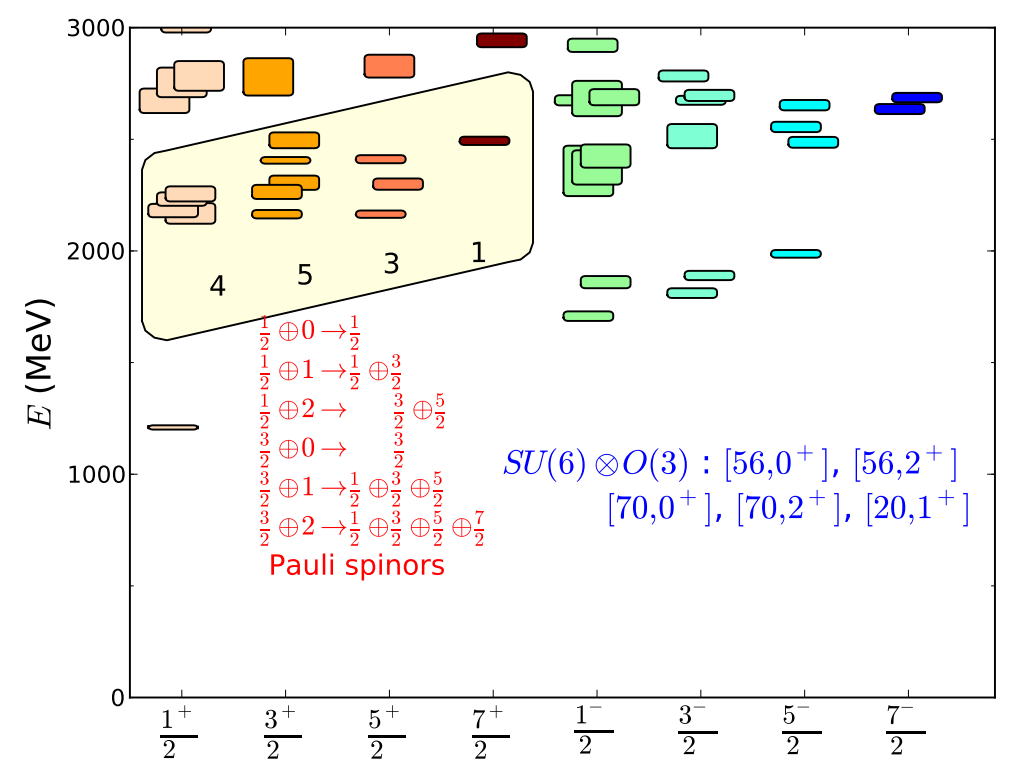

Figure 5: Thirteen $N^{*}$ states corresponding to the $\left[56,0^{+}\right],\left[56,2^{+}\right]\left[70,0^{+}\right]\left[70,0^{+}\right]$and $\left[20,1^{+}\right]$multiplets of $S U(6) \times O(3)$ are shown in the shaded area. The $J$ values and parity are the same as can be formed by $S+L \rightarrow J$, as tabulated in red, when orbital angular momenta and parity $L=0^{+}, 1^{+}$, or $2^{+}$are added to the possible quark spins, providing the same numbers of states of each spin as in the lattice spectrum. 